\title{
Why not test all our genes?
}

\author{
Myles Axton ${ }^{1}$ \\ ${ }^{1}$ Genetics \& Genomics Next Editors
}

May 5, 2020

Gene tests for constitutional ("germline") cancer risk are evolving. We are getting better at finding needles in the haystack, but the haystack is bigger and there are more kinds of needles than we anticipated.

Genes, age and internal and external environment all influence our probability of getting cancer. The rate of lung cancer differs 20-fold by region, globally (Bray et al., 2018), reflecting different rates of tobacco exposure. In contrast, female breast cancer is the most frequently diagnosed cancer in most countries, reflecting less modifiable internal hormonal influences. 5-10\% of these cases arise from heritable causes such as susceptibility to breast cancer and ovarian cancer conferred by cancer susceptibility genes such as BRCA1 and BRCA2. A typical woman's lifetime risk of breast cancer is about $12 \%$, rising to $70 \%$ for a woman carrying one of these mutations (Cline et al., 2019).

\section{A good test is clinically useful}

As gene tests are applied to more genes and people, they are detecting rarer gene variants, those that confer smaller additional risks, and an increasing number of gene variants of unknown significance (VUS). Amid the growing challenges of grading ever more variation in normal human genomes and in genes that contribute to cancer, researchers are using many different strategies to improve the positive predictive value (PPV) of the tests. PPV is the most important measure of clinical utility of a test (Burke, 2014), being the probability that a positive test identifies a variant that actually results in a cancer in that person. Tests can also be clinically useful if they have a high negative predictive value (NPV) being the probability that a negative test results in no cancer in that person. Consequently, the more we know about the gene, genome, heritable cancer predisposition and the person tested, the higher the predictive values and the greater the clinical utility of these tests.

\section{When DNA testing is clinically justified}

Consequently, constitutional DNA testing has been restricted by clinical features of the cancer, or family history associated with high risk of a hereditary causal mutation. But, as larger numbers have been tested, it is possible to use clinical and research data (Lek et al., 2016) together to show where the panels and criteria might be usefully extended. For instance, in one study, 5589 women with breast cancer (but not ovarian cancer) meeting genetic testing criteria for high risk breast and ovarian cancer (GC-HBOC) were tested for a panel of 8 cancer-causing genes (Hauke et al., 2018). In this group, $17 \%$ were expected to carry BRCA1 or BRCA2 mutations. 5 other mutated cancer genes were detected in 312 cases, one gene was not associated with breast cancer and two genes require further investigation in a larger cohort. In a second study using a 17-gene panel, pathogenic mutations were found in $12 \%$ of 10,901 women, specifically those diagnosed with the high-risk triple negative subtype of breast cancer previously associated with BRCA1 mutation (Shimelis et al., 2018). Here they indeed found mutations in BRCA1 but also BRCA2. Further, $3.7 \%$ of triple-negative cancer was associated with 3 other high-risk genes (odds ratio $>5$ ) and 3 genes conferring a moderate risk (odds ratio $>2$ ). A third study investigated the performance of a gene panel in all types of female breast cancer (Couch et al., 2017). Among 41,611 women with breast cancer tested with a 21-gene panel usually 
used only for hereditary cancer, $4.02 \%$ carried BRCA1 or BRCA2 mutations, and $6.18 \%$ of the women carried mutations in 5 genes conferring high or moderate risk of breast cancer. 8 genes associated with other cancers were not found associated with increased breast cancer risk.

\section{Known knowns}

Identifying the known knowns is getting more complex. Panels of known high penetrance cancer-causing genes are scrutinized by DNA sequencing in individuals who qualify because of clinical and family history. For each type of cancer there are different criteria, for instance for high risk of hereditary breast-or-ovarian cancer or hereditary non-polyposis colon cancer respectively. However, the set of cancers caused by each mutated gene is broader than initially appreciated (LaDuca et al., 2019), so it may be necessary to broaden the set of clinical features used to justify testing, extend the set of genes on the test panel, or include more individuals in familial testing. Still, it is worth bearing in mind that prediction of the site of any future cancer, or the outcome of an existing tumor becomes more difficult as the complexity of the genetic test and its applications grow.

\section{Known unknowns}

Sorting the known unknowns is difficult enough. Of the 26,375 BRCA1 and BRCA2 variants reported in the public BRCA Exchange over half have no clinical interpretation. In order for experts to assess each of the rare variants, they must be collected from testing centers into databases such as ClinVar and LOVD . The review process entails combining statistical and rules-based information in a bayesian likelihood framework. One such framework has been developed by the ENIGMA consortium (Parsons et al., 2019) to assign interpretations for 7,256 variants in BRCA1 and BRCA2. Predicting clinical impact of a variant remain difficult, even using challenge-assisted computational and statistical workflows to interpret variants based on clinical and genomic information, with the best predictors wrong for about one out of every five variants (Cline et al., 2019).

\section{Unknown unknowns}

Assigning the prevalence and cancer-causing potential of mutations in the best understood genes is beset with unknown unknowns, since only about $30 \%$ of the BRCA1 variants in reported to the ClinVar database and less than $5 \%$ of all possible single amino acid substitutions have ever been seen in the largest collection of over 120,000 whole genome sequences (Karczewski et al., 2019) assembled in the gnomAD database (2,129 BRCA1 variants of the BRCA Exchange variants are also seen in gnomAD). Gene editing allows VUS to be assigned a pathogenicity score by testing all possible substitution mutations for cell survival or cell function before they are discovered in individual people (Findlay et al., 2018). BRCA1 and four other tumor suppressor genes are essential in haploid cell lines. In those, most of 3893 BRCA1 mutations were just like known benign variants that allowed cells. In contrast, 21 known cancer-causing mutations that had no function were lethal, allowing 400 non-functional missense mutations and 300 impairing gene expression to be labeled pathogenic for the first time. Assays testing homology-directed repair of double strand DNA breaks (Starita et al., 2018) can be used to determine the activity of $6 \%$ or so mutations that showed partial loss of function in the viability assay.

\section{Gene-environment effects}

The smaller contributions of many genes throughout the genome are thought to explain population cancer risk, together with environmental factors such as hormone exposure, smoking and aging (Cronin et al., 2018). At the population level, about $18 \%$ of the familial relative risk of breast cancer can currently be explained by mostly common variants identified by genome wide association studies (Michailidou et al., 2017). Using these variants for polygenic prediction, the $1 \%$ of women with the highest predicted genetic risk would have a 3.5 -fold ( $42 \%$ lifetime risk) compared to the population average ( $12 \%$ lifetime risk). Such tests are undergoing clinical investigation for their utility in prioritizing early detection and prevention strategies, particularly for those with sparse or unrecorded family history of cancer. 


\section{References}

Global cancer statistics 2018: GLOBOCAN estimates of incidence and mortality worldwide for 36 cancers in 185 countries. (2018). CA: A Cancer Journal for Clinicians, 68(6), 394-424. https://doi.org/10.3322/ caac. 21492

Assessment of blind predictions of the clinical significance of BRCA1 and BRCA2 variants. (2019). Human Mutation, 40(9), 1546-1556. https://doi.org/10.1002/humu.23861

Genetic Tests: Clinical Validity and Clinical Utility. (2014). Current Protocols in Human Genetics, 81(1). https://doi.org/10.1002/0471142905.hg0915s81

Analysis of protein-coding genetic variation in 60,706 humans. (2016). Nature, 536(7616), 285-291. https: //doi.org/10.1038/nature19057

Gene panel testing of 5589 BRCA1/2 -negative index patients with breast cancer in a routine diagnostic setting: results of the German Consortium for Hereditary Breast and Ovarian Cancer. (2018). Cancer Medicine, 7(4), 1349-1358. https://doi.org/10.1002/cam4.1376

Triple-Negative Breast Cancer Risk Genes Identified by Multigene Hereditary Cancer Panel Testing. (2018). JNCI: Journal of the National Cancer Institute, 110(8), 855-862. https://doi.org/10.1093/jnci/djy106

Associations Between Cancer Predisposition Testing Panel Genes and Breast Cancer. (2017). JAMA Oncology, 3(9), 1190. https://doi.org/10.1001/jamaoncol.2017.0424

A clinical guide to hereditary cancer panel testing: evaluation of gene-specific cancer associations and sensitivity of genetic testing criteria in a cohort of 165,000 high-risk patients. (2019). Genetics in Medicine. https://doi.org/10.1038/s41436-019-0633-8

Large scale multifactorial likelihood quantitative analysis of BRCA1 and BRCA2 variants: An ENIGMA resource to support clinical variant classification. (2019). Human Mutation, 40(9), 1557-1578. https: //doi.org/10.1002/humu.23818

Variation across 141,456 human exomes and genomes reveals the spectrum of loss-of-function intolerance across human protein-coding genes. (2019). https://doi.org/10.1101/531210

Accurate classification of BRCA1 variants with saturation genome editing. (2018). Nature, 562(7726), 217-222. https://doi.org/10.1038/s41586-018-0461-z

A Multiplex Homology-Directed DNA Repair Assay Reveals the Impact of More Than 1,000 BRCA1 Missense Substitution Variants on Protein Function. (2018). The American Journal of Human Genetics, 103(4), 498-508. https://doi.org/10.1016/j.ajhg.2018.07.016

Annual Report to the Nation on the Status of Cancer part I: National cancer statistics. (2018). Cancer, 124(13), 2785-2800. https://doi.org/10.1002/cncr.31551

Association analysis identifies 65 new breast cancer risk loci. (2017). Nature, 551(7678), 92-94. https: //doi.org/10.1038/nature24284 